\title{
Importance of labelling and patient knowledge to ensure proper care during drug dispensing: A case study from a tertiary hospital in Ethiopia
}

\author{
Seblework Mekonen ${ }^{1 *}$, Wondimu Samuel Manalew ${ }^{2}$, Argaw Ambelu $^{1}$ \\ ${ }^{1}$ Department of Environmental Health Sciences \& Technology, Jimma University, Jimma, Ethiopia; \\ *Corresponding Author: seblework2001@yahoo.com, seblework.mekonen@ju.edu.et \\ ${ }^{2}$ Graduate Program in Economic Development, Vanderbilt University, Nashville, USA
}

Received 9 July 2013; revised 2 December 2013; accepted 15 December 2013

Copyright (c) 2014 Seblework Mekonen et al. This is an open access article distributed under the Creative Commons Attribution License, which permits unrestricted use, distribution, and reproduction in any medium, provided the original work is properly cited. In accordance of the Creative Commons Attribution License all Copyrights (C) 2014 are reserved for SCIRP and the owner of the intellectual property Seblework Mekonen et al. All Copyright (C) 2014 are guarded by law and by SCIRP as a guardian.

\section{ABSTRACT}

Correct drug labelling is central for ensuring proper drug dispensing and thus for patient safety. Labelling errors may result in adverse health outcomes. The objective of this study was, therefore, to assess the effect of labelling on the quality of drug dispensing and patient knowledge about dispensed drugs in Jimma University model and specialized hospital outpatient Pharmacies. Individual packages with prescribed drugs were examined using pretested questionnaire and observational check lists during the dispensing process. Patients' knowledge about drugs dispensed to them was assessed at the exit interview using a pretested questionnaire. Out of 743 prescribed drugs, 682 (91.8\%) were dispensed to 426 patients. The average labelling score (range from 0 to 6 ) of dispensed drugs in Model and Outpatient pharmacy was 2.00 (95\% $\mathrm{Cl} 1.97$ to 2.04$)$ and $1.73(95 \% \mathrm{Cl} 1.6$ to 1.8$)$ respectively, with overall average labelling score of 1.90 (95\% Cl 1.84 to 1.91). The average patient knowledge score (range from 0 t0 5) was 3.45 (95\% $\mathrm{Cl} 3.31$ to 3.59$)$ and 3.5 (95\% Cl 3.35 to 3.64$)$ for model and outpatient pharmacy, respectively, while the overall average knowledge score was 3.46 (95\% Cl 3.37 to 3.57 ). Major labelling problems were absence of patient's name and dose followed by frequency of administration, duration of treatment, and the reason for prescription. Literacy status of patients had a significant effect on their knowledge $(p<0.005)$ but age had not $(p>0.05)$. We recommend that corrective measures targeting both, labelling and patients' knowledge should be implemented to improve the patients' safety and drug therapy adherence.

\section{KEYWORDS}

Labelling; Patient Knowledge; Patient Care; Outpatient Pharmacy; Dispensed Drug

\section{INTRODUCTION}

Rational prescription and drug use have been a concern in both developed and developing countries during the last two decades and have been promoted by World Health Organization WHO and others. Within drug use programmes, efforts have often been concentrated on ensuring rational prescribing habits. The quality of dispensing and patient knowledge of drugs has often been overlooked [1-4].

The primary purpose of labelling is to provide all the necessary information needed to correct dispensing of a prescribed drug. The label gives patients the relevant information which they need for proper use of the dispensed drug. In addition to proper labelling, patients' knowledge about the prescribed drug is an essential prerequisite for patient compliance [5,6]. Any error or failure in the dispensing process can jeopardize the care of the patient [7]. Dispensing is a critical part of the drug use process and plays an important role in avoiding irrational drug use. The manner in which drugs are taken by patients is often influenced by the dispensing protocol and information given during the dispensing process $[8,9]$. All patients are potentially at risk for medication 
errors. A recent study from the US hospitals has shown that $55 \%$ of the total hospital medication errors involved older adults [10]. The potential for medication errors increases as the number of drugs administered increases. Individuals aged 65 years and older use three times the number of medication than individuals in younger age groups. These medication errors can be prevented by correct and proper labelling, and giving patient appropriate drug information during dispensing [11].

The time spent with the patient by a dispenser is a good indicator of the service quality received by the patient. Inappropriate dispensing and use of drugs can lead to ineffective health care. Studies conducted in both developed and developing countries [12-14] indicate that incorrect dispensing, self medication and the use of subtherapeutic doses are major cases of irrational drug use.

\section{MATERIAL AND METHODS}

\subsection{Study Site}

The study was conducted at Jimma University located in Jimma town, approximately $355 \mathrm{~km}$ from the capital, Addis Ababa. The University's college of Public Health and Medical Sciences comprises of 18 departments which train medical and paramedical students. Among the facilities available, the university's Model Pharmacy which serves as a training centre for pharmacy students exists. In addition, the pharmacy also provides service to hospital patients and serves as a model for other private and government pharmacies in terms of drug availability and quality of service. In addition to the Model Pharmacy, The Jimma University Specialized Hospital (JUSH) also has four other pharmacy units namely: inpatient, outpatient, antiretroviral therapy (ART), and pharmacy store. One pharmacist and five technicians were on duty in the four units at the time of data collection (January to March 2007).

\subsection{Study Design and Data Collection}

Single institution based cross sectional study design was used for assessment of individual drug labelling using previously prepared and pre-tested check list.

In addition, participative observations of the dispensing process were conducted. Patients' knowledge of dispensed drugs and understanding of the information provided were assessed at the exit interview. The drug labelling quality was assessed by calculating mean labelling scores composed of six dispensing fields: 1) name of a patient, 2) drug name, 3) drug strength, 4) drug dose, 5) frequency of drug administration, and 6) duration of treatment. Correct response to each field was assigned a score " 1 ". Incorrect or absent information was assigned a score " 0 " (creating a score range from 0 to 6). Each pa- tient's knowledge about dispensed drug(s) was obtained at the exit from the pharmacy after dispensing. The patient recall of name and dose of a drug, frequency of administration, duration of treatment, and reason for prescription (incorrect recall $=0,1$ point for each correct recall attribute; maximum score $=5$ ).

\subsection{Data Analysis}

Data was compiled and analysed using SPSS for Windows Version 16. Correlation coefficients and Chisquare tests were used to determine the relationship between dependent and predictor variables.

\subsection{Ethical Consideration}

The research proposal was reviewed and approved by Ethical Review Board of the Jimma University prior to conducting the study. Moreover, data was collected after consent was obtained from each study unit (patient or care giver).

\section{RESULTS}

A total of 426 patients, 220 from model pharmacy, and 206 from outpatient pharmacy were included in the study.

\subsection{Socio-Demographic Characteristics}

Among 426 patients, 262 (61.5\%) and 164 (38.5\%) were males and females, respectively. The majority was within age group of 18 - 44 years old and 45 - 65 years of age. Most of patients (79.9\%) were literate at different educational levels and $20.9 \%$ were illiterate.

\subsection{Dispensed Drugs}

Total of 743 drugs were prescribed to be dispensed to 426 patients and 682 (91.8\%) were dispensed. Since 2 drugs per prescription per patients were considered, 58 drugs were eliminated, leaving 624 drugs for the assessment of labelling patterns and patients knowledge analyses. In the model and outpatient pharmacies, 366 and 377 drugs were prescribed to 220 and 206 patients, respectively. From the prescribed drugs, 349 (95.4\%) and 333 (88.3\%) drugs were dispensed in the Model and Outpatient pharmacies, respectively.

Majority (53.5\%) of the patients were dispensed one drug, $35.4 \%$ two drugs, and $11 \%$ were dispensed more than two drugs per prescription (Figure 1).

Major class of drugs dispensed to patients (Table 1) were anti-infective (40\%) followed by non-steroidal antiinflammatory drugs (NSAID) (15\%), central nervous system (CNS) drugs (13\%), cardio vascularsystem (CVS) drugs (12\%), and gastro intestinal tract (GIT) drugs 38 
Table 1. Labelling score of dispensed drugs in model and outpatient pharmacies.

\begin{tabular}{|c|c|c|c|c|c|c|c|c|c|c|}
\hline & \multicolumn{7}{|c|}{ Labelling score } & \multirow{2}{*}{ Total } & \multirow{2}{*}{ Average labelling score } & \multirow{2}{*}{ Test statistics } \\
\hline & 0 & 1 & 2 & 3 & 4 & 5 & 6 & & & \\
\hline Anti infective & 2 & 21 & 221 & 3 & 0 & 0 & 0 & 247 & 1.91 & \multirow{7}{*}{$\begin{array}{c}\text { Chi-square test }=91.9 \\
\text { p-value } \leq 0.005\end{array}$} \\
\hline NSAIDs & 0 & 0 & 92 & 1 & 0 & 0 & 0 & 93 & 2.01 & \\
\hline CNS drugs & 5 & 24 & 53 & 0 & 2 & 0 & 0 & 84 & 1.64 & \\
\hline GIT drugs & 0 & 0 & 38 & 0 & 0 & 0 & 0 & 38 & 2.0 & \\
\hline CVS drugs & 2 & 16 & 55 & 1 & 0 & 0 & 0 & 74 & 1.74 & \\
\hline Others & 0 & 10 & 74 & 4 & 0 & 0 & 0 & 88 & 1.93 & \\
\hline Total drugs & 9 & 71 & 533 & 9 & 2 & 0 & 0 & 624 & 11.23 & \\
\hline
\end{tabular}

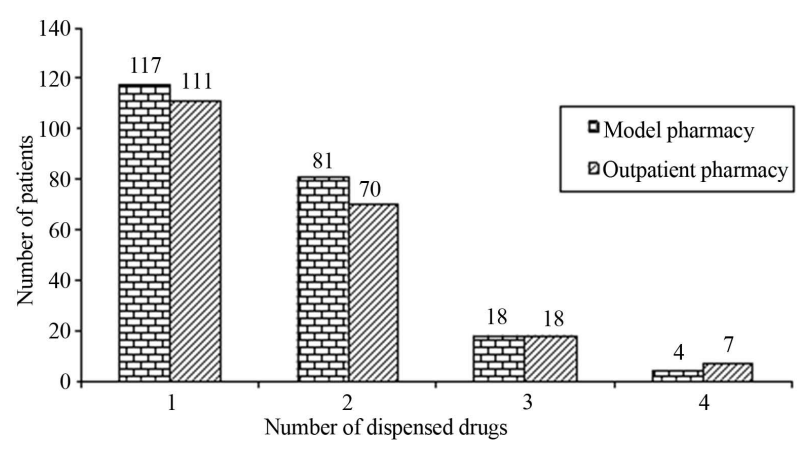

Figure 1. Number of drugs dispensed per encounter in the model and outpatient pharmacies.

(6\%). The "other" class (14\%) included vitamins, oral rehydration salt, and glucose. (Figure 2)

\subsection{Labelling Pattern and Score}

Most of the dispensed drugs, 5 (85.3\%), in both pharmacies had a labelling scores of 2 and there was no drug dispensed with the score 5 or 6The average labelling score of dispensed drugs in model and outpatient pharmacies was 2.00 (95\% confidence interval [CI]: 1.97 2.04) and 1.73 (95\% CI: 1.6 - 1.8), respectively. And overall average labelling score was 1.9 Labelling scores for different drug classes of drugs from both pharmacies ranged from 1.64 (CNS drugs) to 2.01 (NSIDs) (Table 1).

Of all dispensed drugs from the two pharmacies no label was found with patient's name. Majority (83\%) of the drugs were dispensed as original packs which have label of name and strength of the drug and the rest have few labels of dispensing attributes (Figure 3).

\subsection{Patient Knowledge}

The name of drug was recalled in 138 (22.1\%), dose in 533 (85.4\%), frequency of administration in 539 (86.4\%), duration of treatment in 477 (76.4\%), and the reason for

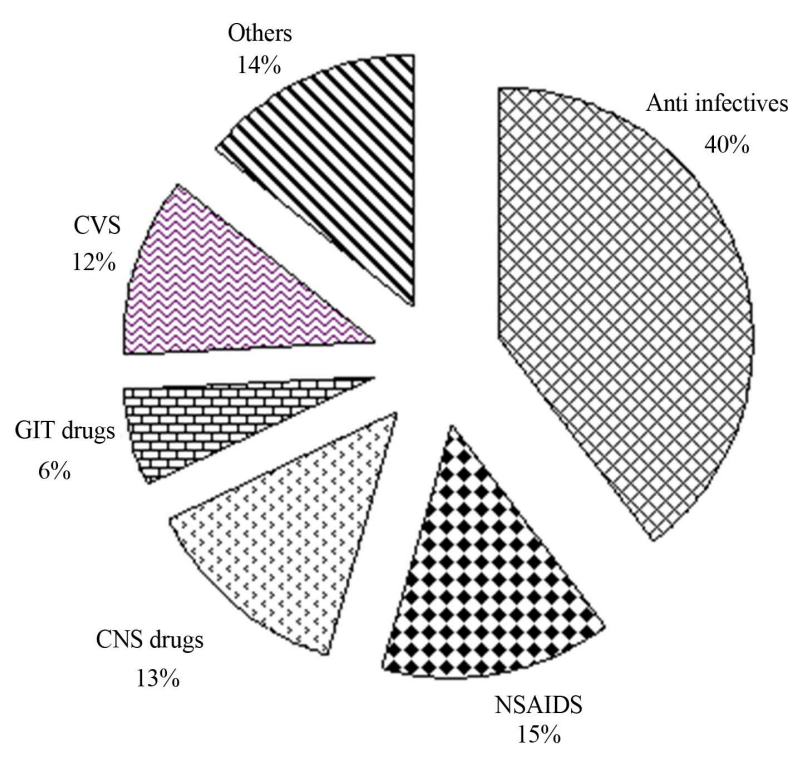

Figure 2. Class of drugs dispensed to patients in model and outpatient pharmacies.

prescription in 471 (75.5\%) of dispensed drugs. The average knowledge scores of patients who took drugs from model and outpatient pharmacies were 3.45 and 3.5, respectively. The result showed that the average knowledge score of patients from both pharmacies is 3.46.

From all the knowledge attributes "name of drug" was recalled by least number of patients in both pharmacies. However, other knowledge attributes of most drugs dispensed were recalled by majority of the patients (Figure 4). The literacy status of patients who recalled most of the knowledge attributes to dispensed drugs were calculated and the least knowledge score was found for those who have informal education and illiterates (2.88 and 3.08 , respectively).

The highest knowledge score was found for patients whose literacy status was 9 to 10 grades ( 4 on 5 scales). But majority of the patients in both literacy groups did not recall the name of dispensed drugs. 


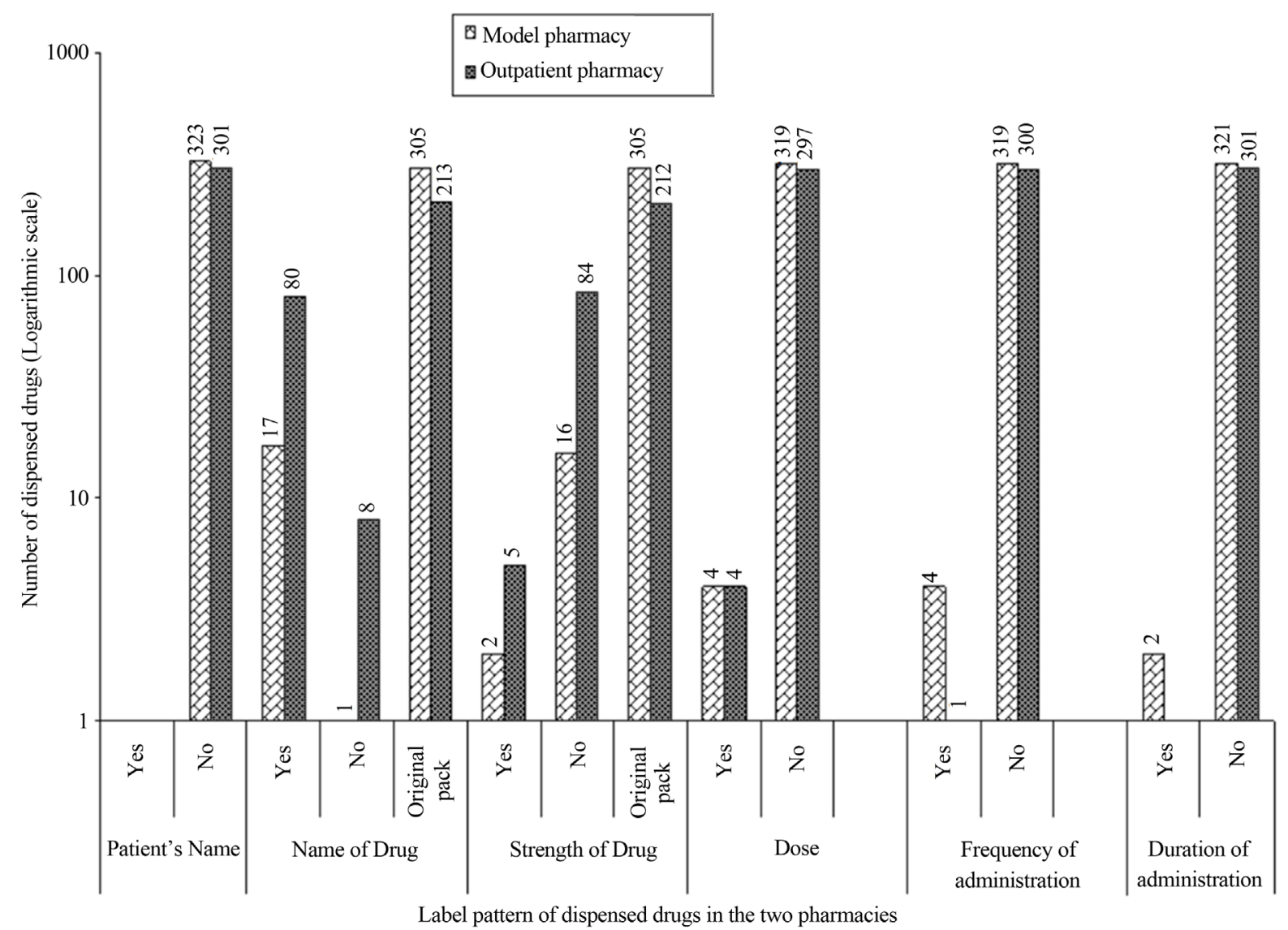

Figure 3. Labelling pattern of dispensed drugs in model and outpatient pharmacies.

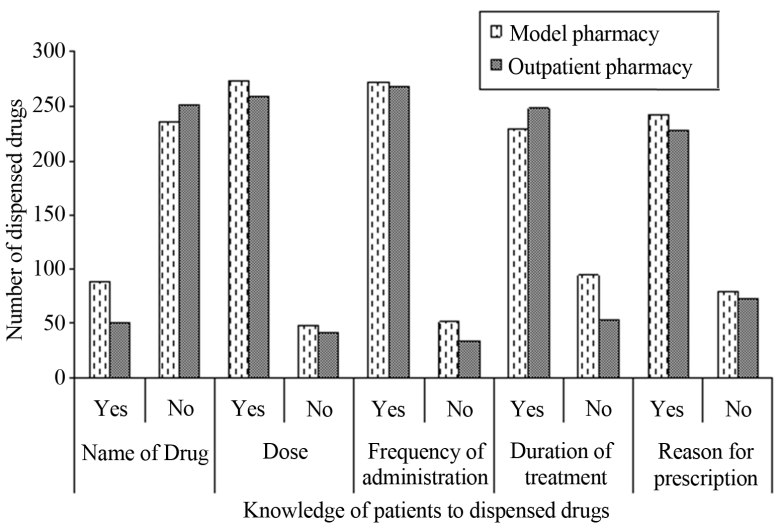

Figure 4. Response of patients to the knowledge of dispensing attributes in model and outpatient pharmacies, Jimma.

\section{DISCUSSION}

In order to understand the way drugs are used, it is important to consider what takes place at the health facilities from both the provider's and the patient's perspectives. Patients, for whom pharmaceuticals are prescribed, should at a minimum, receive well labelled medications, and should understand how to take each drug [4].
This study has shown that one of most important dispensing attribute, patient's name, was not labelled in any of the dispensed drugs. This may result in confusion of medication between family members, or other groups of people. In prisons and other institutions where people reside in large groups, it is highly likely that individuals take the drugs which are actually not prescribed to them. The drugs dispensed at both pharmacies have overall average labelling score of 1.9 on 6 scales (representing $32 \%$ of total score).

Knowledge scores of patients to dispensed drugs by age were also tabulated and higher score (3.94) was observed in $10-17$ years of age group. The least score (3.09) was found in patients with age group of above 65 (Table 2).

The average dispensing time spent to individual patients in model and outpatient pharmacies was 125.5 and 39.3 seconds, respectively and the average dispensing counselling time was 14.4 and 19.8 seconds. The maximum and minimum values to each pharmacy have wider gaps.

Pattern between these pharmacies is significantly different $(\mathrm{p}<0.005)$. This may be due to less number of druggists involved in dispensing at outpatient pharmacy. 
Table 2. Knowledge score of different age groups and literacy status of patients to different dispensing attributes of dispensed drugs at model and outpatient pharmacies, Jimma.

\begin{tabular}{|c|c|c|c|c|c|c|c|c|c|c|}
\hline & & \multicolumn{6}{|c|}{ Knowledge scores } & \multirow{2}{*}{ Total } & \multirow{2}{*}{$\begin{array}{l}\text { Average } \\
\text { knowledge } \\
\text { Score }\end{array}$} & \multirow{2}{*}{ Statistical test } \\
\hline & & 0 & 1 & 2 & 3 & 4 & 5 & & & \\
\hline \multirow{4}{*}{ Age group } & $10-17$ yrs & 0 & 0 & 1 & 7 & 21 & 7 & 36 & 3.94 & \multirow{4}{*}{$\begin{array}{c}\text { Chi-square test }=14.9 \\
\text { p-value }>0.05\end{array}$} \\
\hline & $18-44$ yrs & 25 & 22 & 31 & 104 & 189 & 79 & 450 & 3.44 & \\
\hline & $45-65$ yrs & 3 & 3 & 11 & 27 & 56 & 16 & 116 & 3.53 & \\
\hline & $65+$ & 2 & 1 & 2 & 6 & 10 & 1 & 22 & 3.09 & \\
\hline \multirow{7}{*}{ Educational status } & Illiterate & 10 & 9 & 12 & 34 & 61 & 5 & 131 & 3.08 & \multirow{7}{*}{$\begin{array}{c}\text { Chi Square test }=74 \\
\text { p-value }<0.005\end{array}$} \\
\hline & $1-4$ & 2 & 2 & 3 & 12 & 31 & 10 & 60 & 3.63 & \\
\hline & $5-8$ & 6 & 7 & 13 & 26 & 62 & 14 & 128 & 3.35 & \\
\hline & $9-10$ & 3 & 3 & 4 & 10 & 17 & 9 & 46 & 4.00 & \\
\hline & $10+$ & 2 & 0 & 1 & 9 & 25 & 8 & 45 & 3.76 & \\
\hline & $12+$ & 4 & 2 & 8 & 44 & 67 & 56 & 181 & 3.86 & \\
\hline & $99^{*}$ & 3 & 3 & 4 & 9 & 13 & 1 & 33 & 2.88 & \\
\hline
\end{tabular}

Only one druggist was in charge for the dispensing process during study period. The work over load obviously reduces the efficiency at which quality service is provided to patients. Similar problems have been reported in USA by Mitchener and David [15] that too much prescription and few numbers of pharmacists involved in pharmacies has resulted in medication errors.

This shows that there are nearly two labels on each of the dispensed drugs. The score would even be lesser if there were no original pack dispensed drugs (83\%) which have actually two labels, name and strength of the drug. This score is by far less than the labelling score of dispensed drugs studied in Botswana which is 2.75 on 5 scale (representing $55 \%$ of total score) [6].

At facility level, the average labelling scores at model and outpatient pharmacies were 2.0 and 1.8, respectively. Statistical tests showed that labelling

Significant variation of labelling scores were also observed by major class of dispensed drugs $(\mathrm{p}<0.005)$. The perception that professionals involved in dispensing have towards patient's knowledge might have also affected the result. For CVS and CNS drugs, for example, professionals usually assume that the patient knows the correct dosage as the drugs are normally taken on a regular basis relatively for a long period.

At some point during examination or dispensing process, details about the medication prescribed are often explained to the patient. Similarly, this study included five dispensing attributes, namely: name and dose of drug, frequency of administration, duration of treatment, and the reason for prescription to assess the understanding of patient to the information provided. However, evaluating the patient's knowledge of when and in what quantity each drug should be taken would be simple for measurement.

Accordingly, dose and frequency of administration were recalled in $85.4 \%$ and $86.4 \%$ for the drugs dispensed, respectively. The least (22\%) recalled attribute of dispensed drug was "name of drug". This is probably due to the trend that professionals involved in dispensing do not usually tell the names of drugs to patients. In fact, "drug name" is one of the most important indicator of patient knowledge as patients who know drug names can make proper use of the drugs without expecting much help from the health professionals. Patient's knowledge of drug's name would also make retreatment with different drugs easier in case the drug first prescribed is found ineffective or hypersensitive. Patient's knowledge helps the health professionals in determining alternative drugs during patient's revisit. In this study majority of patients have good knowledge to other attributes of dispensed drugs. The average knowledge score of patients in model and Outpatient pharmacies were 3.42 and 3.5, respectively, while the overall average knowledge score was 3.46 (representing $69.2 \%$ of total score) which is better score than the value obtained in Botswana (2.5 on 4 scale, representing $63 \%$ of total scores) [6].

Patients who recalled the correct dose of the dispensed drugs in both model and Outpatient pharmacies were about $85 \%$. This value is higher than the results found in Yugoslavia which is $68 \%$ of the total dispensed [16]. The elevated knowledge score in this study compared to other studies conducted elsewhere $[6,16]$ may be due to the literacy status of the patients taking the drug. Approxi- 
mately, $80 \%$ of the patients were literate at different levels. In addition, majority of the patients (53.5\%) were taking only one drug which can make them easily understand the information given to them. Patients at Outpatient pharmacy had higher knowledge scores than in Model pharmacy. A plausible explanation is that most of the patients served there were chronically ill who, due to prolonged use of drugs, became familiar with the drugs taken.

Age of patients did not have any significant association ( $p>0.05$ ) in recalling the dispensing attributes. This might be due to most of the patients who took dispensed drugs were within age groups of 18 to 65 years old and they easily understand the information what the dispenser provided during dispensing process.

The number of drugs actually dispensed for the total patients were $682(91.8 \%)$ out of 743 prescribed drugs. This result is better as compared to the drugs actually dispensed in Jordan (81.8\%) [17] and similar to findings of preliminary survey on drug dispensing and labelling pattern in hospitals of Southern Nations and Nationalities Peoples Region (SNNPR), Ethiopia, (90\%) [18]. This may be due to better availability of drugs in the two pharmacies since they are model and teaching pharmacies.

The average dispensing time in the two pharmacies was 82.4 seconds. At facility level, the average dispensing time of model and outpatient pharmacies have 125.5 and 39.3 seconds. The average dispensing time obtained from model pharmacy is higher than the average dispensing time found in the study done at hospitals of SNNPR (70 sec) [18], and Health Stations (90 sec.) and Health Centres (114 sec.) of Northern Ethiopia, Gondar [19]. Similarly, it is also has higher dispensing time than the time found from Jordan (28.8 sec) [17]; Yugoslavia (20.5 - 48.2 sec.) [16]; and Tanzania (78 sec.) [13]. The higher average dispensing time at model pharmacy doesn't mean that more information about dispensed drugs was given to the patients. This can be verified by the better knowledge score and dispensing counselling time obtained in outpatient pharmacy. The higher dispensing time observed at model pharmacy is due to the inclusiveness of the time spent for cost estimation of the drug and paying the drug charge. But in outpatient pharmacy, the patients are taking drugs free of charge in that case no time is spent for estimation and payment. The average dispensing time of both pharmacies is within the range of international standard (13 - 86 seconds) [4]. To the contrary higher average dispensing counselling time is observed in outpatient than model pharmacy (19.8 and 14.4 sec., respectively). This time is exclusively the time spent by the dispenser with the patient during drug dispensing and counselling. The higher dispensing counselling time in the outpatient pharmacy may be due to the delivery of more than one drug for a patient. This dispensing counselling time found in both pharmacies is much lower than the time given to patients for advising and giving information about dispensed drugs per patient (189 sec.) in South Africa [20].

Several study variables were significantly correlated $(p<0.01)$ to one another. For example, there is big difference of labelling of the name of the drug on dispensed drugs between the two pharmacies. The probability of dispensing drugs without the name of the drug was higher in outpatient than the model pharmacy. Similarly, inverse relationship was observed for overall labelling score between the two pharmacies $(r=-0.83)$. Patient's literacy affected their ability to recall name and dose of the dispensed drugs. This suggests that effort should be made at the community level to help patients to improve their understanding attributes about dispensed drugs.

\section{CONCLUSIONS}

Inadequate labelling of dispensed drugs to patients was the major problem observed in both of the pharmacies examined. Although, most of the dispensed drugs were original packed, they were not adequately labelled and lacked the other four drug label attributes. The other main problem of the labelling pattern was absence of patients' names in all of the dispensed drugs. These inconveniencies could curtail the health services of pharmacies, which may lead to irrational drug use. Educational status of patients can be a good indicator of better knowledge on dispensed drugs. Longer average dispensing time does not necessarily mean that proper information is provided to the patient. Average dispensing counselling time which is directly spent with the patients is much better quality indicator.

We recommend that corrective measures targeting both, labelling and patients' knowledge should be implemented to improve the patients' safety and drug therapy adherence.

\section{ACKNOWLEDGMENT}

The authors are grateful to Jimma University for financially supporting the study. We are also grateful to pharmacy students of the university who have helped us with data collection.

\section{CONFLICT OF INTEREST}

Authors do not have any conflict of interest arising from this study.

\section{REFERENCES}

[1] World Health Organization (WHO). (1987) The rational use of drugs. Conference of Experts on the Rational Use of Drugs, Nairobi, 25-29 November 1985. 
[2] Laing, R.O. (1990) Rational drug use: An unsolved problem. Tropical Doctor, 20, 101-103.

[3] Dukes, M.N.G. (1993) Drug utilization studies. Methods and uses. WHO Regional Publications, European Series No. 45. Copenhagen.

[4] World Health Organization. (1997) Managing drug supply. 2nd Edition, Kumarian Press, West Hartford.

[5] Smith, F. (2003) Drug use in Sub-Saharan Africa: Quality in processes-Safety in use. Quality and Safety in Health Care, 12, 164-165. http://dx.doi.org/10.1136/qhc.12.3.164

[6] Boonstra, E., Lindbaek, M., Ngome, E., et al. (2003) Labeling and patient knowledge of dispensed drugs as quality indicators in primary care in Botswana. Quality and safe in Health Care, 12, 168-175.

[7] Hogerzeil, H.V., Ross-Degnan, B.D., Laing, R.O., et al. (1993) Field-tests for rational drug use in twelve developing countries. Lancet, 342, 1408-1410. http://dx.doi.org/10.1016/0140-6736(93)92760-Q

[8] Margaret-Mary, A. and David, O.A. (1997) The impact of three forms of educational interventions on dispensing practices. International Conferences on Improving Use of Medicines (ICIUM).

http://mednet3.who.int/icium/icium1997/posters/2b1 txt1 .html

[9] Agency for Health Care Research and Quality (AHCQ). (2003) One third of elderly home health care patients are having problems with their medication. http://www.ahrq.gov/research/sepol

[10] US Pharmacopial. (2003) USP releases fourth annual report on medication errors in US hospitals. US Pharmacopial, Rockville.

[11] Liu, G.G. and Christensen, D.B. (2002) The continuing challenge of inappropriate prescribing in the elderly: An update of the evidence. Journal of American Pharmacists Association, 42, 847-857. http://dx.doi.org/10.1331/108658002762063682

[12] World Health Organization (WHO). (1993) How to in- vestigate drug use in heath facilities. Selected drug use indicators. World Health Organization, Geneva.

[13] Oferi-Adjei, D. (1992) Report on Tanzanian field test. INRUD News, 3.

[14] Massele, A.Y., Nsimba, S.E. and Rimoy, G. (2001) Prescribing habits in church-owned primary health care facilities in Dar es Salaam and other Tanzanian coast regions. East African Medical Journal, 78, 510-514. http://dx.doi.org/10.4314/eamj.v78i10.8958

[15] Schillinger, D., Machtinger, E., Wang, F., Rodriguez, M., Bindman, A. (2005) Preventing medication errors in am-bulatory care: The importance of establishing regimen concordance. In: Henriksen, K., Battles, J.B., Marks, E.S and Lewin, D.I., Eds., Advances in Patient Safety Research Implement Vol. 1 Research Findings, Agency for Healthcare Research and Quality, Rockville.

[16] Jankovic, S.M., Maksimovic, M.R., Vusanovic, A., Kostic, I.R., Kovacevic, Z.N. and Mitric, M. (2001) Service quality in public and private pharmacies in the city of Kragujevac, Yugoslavia. Croatian Medical Journal, 42, 88-91.

[17] Otoom, S., Batieha, A., Hadidi, H., Hasan, M. and AlSaudi, K. (2002) Evaluation of drug use in Jordan using WHO patient care and health facility indicators. Eastern Mediterranean Health Hournal, 8, 544-549.

[18] Dawit, D., Zeleke, G. and Shiferaw, T.M. (1998) A preliminary survey on drug dispensing pattern in some hospitals in Southern Nations, Nationalities and Peoples Region, Ethiopia. Communications, Ethiopian Pharmaceutical Journal, 16, 59-63.

[19] Desta, Z., Abula, T., Beyene, L., Fantahun, M., Yohannes, A.G. and Ayalew, S. (1997) Evaluation of rational drug use and prescribing in primary health care facilities in Northwestern Ethiopia. East African Medical Journal, 74, 758-763.

[20] Truter, I. (2006) Dispensing service research-pilot project. Pharmacia-Official Publication of the South African Pharmacy Council, 14, 2. 